\title{
Transformation-Optics Description of Nonlocal Effects in Plasmonic Nanostructures
}

\author{
A. I. Fernández-Domínguez, ${ }^{1}$ A. Wiener, ${ }^{1}$ F. J. García-Vidal, ${ }^{2}$ S. A. Maier, ${ }^{1}$ and J. B. Pendry ${ }^{1}$ \\ ${ }^{1}$ The Blackett Laboratory, Department of Physics, Imperial College London, London SW7 2AZ, United Kingdom \\ ${ }^{2}$ Departamento de Física Teórica de la Materia Condensada, Universidad Autónoma de Madrid, E-28049 Madrid, Spain
}

(Received 16 December 2011; published 6 March 2012)

\begin{abstract}
We develop an insightful transformation-optics approach to investigate the impact that nonlocality has on the optical properties of plasmonic nanostructures. The light-harvesting performance of a dimer of touching nanowires is studied by using the hydrodynamical Drude model, which reveals nonlocal resonances not predicted by previous local calculations. Our method clarifies the interplay between radiative and nonlocal effects in this nanoparticle configuration, which enables us to elucidate the optimum size that maximizes its absorption and field enhancement capabilities.
\end{abstract}

DOI: 10.1103/PhysRevLett.108.106802

The boost experienced by plasmonics research [1] during the past decade has been motivated by the ability of surface plasmon polaritons (SPPs) to collect and concentrate light into subwavelength volumes with extreme efficiency. The exploitation of this distinctive SPP attribute in smaller length scales has driven the continuous improvement of nanofabrication and characterization techniques. This miniaturization trend is currently approaching the limit in which the spatial extent of the oscillating electromagnetic (EM) fields is comparable to the Coulomb screening length in noble metals $\left(\delta_{C} \sim 0.1 \mathrm{~nm}\right.$ for $\mathrm{Ag}$ [2]). From a theoretical perspective, the exploration of EM phenomena in this subnanometer regime requires the implementation of nonlocal, spatially dispersive, permittivities that take into account the effect of electron-electron interactions in the dielectric response of metallic nanostructures.

Nonlocal effects in the optical properties of metals have attracted much theoretical interest in the past [3]. More recently, they have been revisited in the context of nanoparticle plasmon resonances using modern computational techniques [4-6]. However, except for a few simple geometries [7,8], an analytical treatment of this fundamental problem is still lacking. In this Letter, we fill this gap and shed light into the impact that spatial dispersion has on plasmonic devices. We present a transformation-optics (TO) approach, inspired by previous (local) studies [9], that incorporates nonlocality into the description of the interaction of light with singular nanoparticle geometries. Specifically, we investigate how the prominent field enhancement capabilities reported for a dimer of touching nanowires are modified once the spatially dispersive character of the metal permittivity is considered. Finally, we explore the interplay between radiative losses and nonlocality in this structure and provide an optimum size for which its light-harvesting capabilities are maximized.

Figure 1(a) depicts the system under study, a pair of touching nanowires of radius $R$ illuminated by a plane wave polarized along the dimer axis. The metal permittivity
PACS numbers: 73.20.Mf, 42.25.Bs, 42.70.-a, 78.67.Bf

is modeled by using the so-called hydrodynamical Drude model [10], which yields $\epsilon_{T}(\omega)=\epsilon_{\infty}\left[1-\frac{\omega_{P}^{2}}{\omega(\omega+i \gamma)}\right]$ and $\epsilon_{L}(\mathbf{k}, \omega)=\epsilon_{\infty}\left[1-\frac{\omega_{P}^{2}}{\omega(\omega+i \gamma)-\beta^{2}|\mathbf{k}|^{2}}\right]$ for transverse and longitudinal EM fields, respectively. The usual Drude constants are obtained from the fitting to experimental data for silver [11], whereas $\beta$, which measures the degree of

(a)
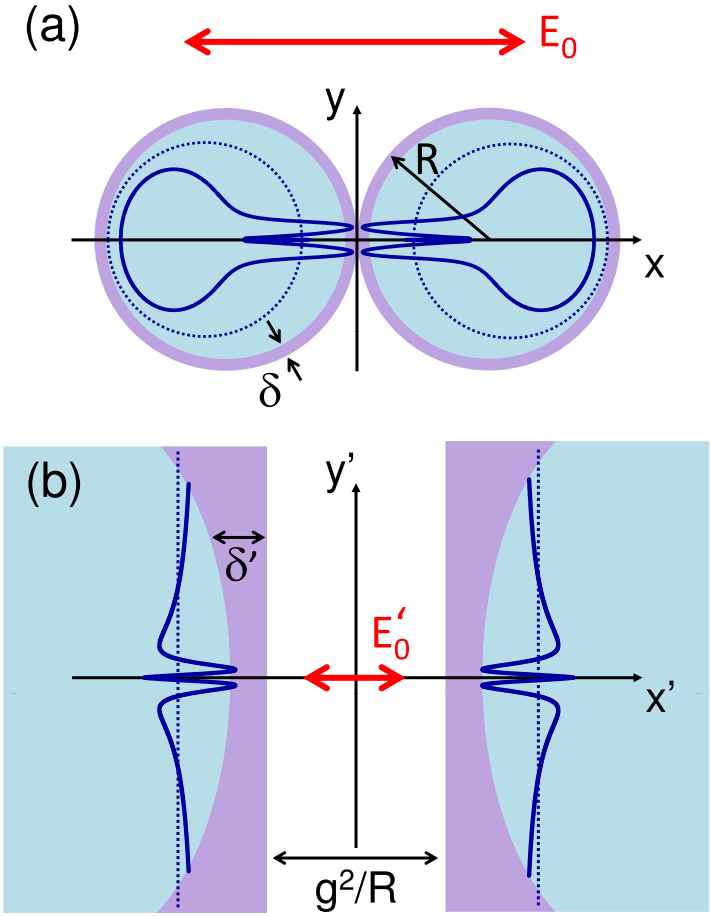

FIG. 1 (color online). Panel (a) shows the problem under study: the nonlocal description of the interaction of light with a dimer of touching metal nanowires. Panel (b) depicts the geometry resulting from the inversion of the original structure. The shaded areas in both panels represent the decay length of the longitudinal plasmons excited in the system. Blue solid lines show artistic plots of the electrostatic potential describing propagating SPPs in both frames. 
nonlocality, is set as a free parameter for the moment. The dark ring in Fig. 1(a) represents the decay length of the longitudinal plasmons into the metal, $\delta$, whose optical excitation is possible due to spatial dispersion. This magnitude reflects the nonvanishing extension of the surface charges induced in the nanowires by the incident light. For frequencies well below $\omega_{P}, \delta=\frac{\beta}{\omega_{P}}$, which shows explicitly the link between the surface charge thickness and the hydrodynamical parameter $\beta$.

Through a conformal inversion of the form $z^{\prime}=\frac{g^{2}}{z^{*}}$, our TO treatment of the problem maps the touching nanowires into the metal-vacuum-metal geometry shown in Fig. 1(b). Note that the operation also converts the incident fields into a dipole source at the intermediate layer. Whereas $\epsilon_{T}(\omega)$ is preserved under the transformation, the $k$ dependence of $\epsilon_{L}(\mathbf{k}, \omega)$ makes it sensitive to the inversion. Assuming that the nonlocal length scale is much smaller than the spatial range in which $\frac{d z^{\prime}}{d z}$ varies significantly, the general relation between the transformed (primed) longitudinal permittivity and its original (unprimed) counterpart reads

$$
\epsilon_{L}^{\prime}\left(\mathbf{k}^{\prime}, \omega\right)=\epsilon_{L}\left(\left|\frac{d z^{\prime}}{d z}\right| \mathbf{k}^{\prime}, \omega\right)
$$

which shows that the only consequence of the mapping is a wave-vector stretching weighted by $\left|\frac{d z^{\prime}}{d z}\right|$. For our inversion, Eq. (1) yields a longitudinal permittivity with $\beta^{\prime}\left(z^{\prime}\right)=\beta \frac{\left|z^{\prime}\right|^{2}}{g^{2}}$, which provides the surface charge thickness a $y^{\prime}$ dependence as sketched in Fig. 1(b).

Maxwell's equations can be solved in the transformed frame under two assumptions. First, we restrict our attention to nanostructure sizes smaller than the incoming wavelength, which enables us to work within the near-field approximation. Importantly, our approach goes beyond the quasistatic limit by describing radiation losses through the radiative reaction concept [12]. The second premise supposes that $\delta^{\prime}\left(y^{\prime}\right)$ varies in space much more slowly than the oscillating EM fields. This makes possible the use of the so-called eikonal (WKB) approximation to describe the propagation of SPP modes along the transformed metal surfaces away from the dipole source. The inclusion of the longitudinal plasmon contribution to the EM fields requires an additional continuity condition at the system boundaries. The appropriate condition for our problem is the continuity of the normal electric field. It arises from the smooth distribution that induced charges acquire in spatially dispersive metal surfaces $[5,8]$. Note that our approach omits electron tunneling effects, which could have some influence in the plasmonic response of metal nanoparticles as well $[13,14]$.

Our TO method yields quasianalytical expressions for the electrostatic potential at each region of the transformed geometry, which can be then mapped into the original frame by using $\phi(z)=\phi^{\prime}\left(z^{\prime}(z)\right)$. Thus, the electric field enhancement at the nanowires surface as a function of the azimuthal angle $\theta$ (see the inset in Fig. 2) can be expressed as

$$
\begin{aligned}
\frac{E_{x}}{E_{0}}= & \frac{-\pi \alpha(\theta) f(\omega)}{(1+\cos \theta) \eta(\omega)}\left[\sin \theta \sinh \frac{\alpha(\theta)}{2}+i \cos \theta \cosh \frac{\alpha(\theta)}{2}\right] \\
& \times\left[\exp \left\{\frac{i}{2} \int_{0}^{\theta} \frac{d \theta^{\prime} \alpha\left(\theta^{\prime}\right)}{1+\cos \theta^{\prime}}\right\}+\exp \left\{\frac{i}{2} \int_{\theta}^{2 \pi} \frac{d \theta^{\prime} \alpha\left(\theta^{\prime}\right)}{1+\cos \theta^{\prime}}\right\}\right],
\end{aligned}
$$

where

$$
\alpha(\theta)=\ln \left|\frac{\epsilon_{T}(\omega)-1}{\epsilon_{T}(\omega) \frac{\gamma(\theta)+1}{\gamma(\theta)-1}+1}\right|
$$

gives the SPP tangential wave vector in the near-field approximation and $\gamma(\theta)=$ $\sqrt{1+4\left[\left(\omega_{P}^{2}-\omega^{2}\right) / \beta^{2} \alpha^{2}(\theta)\right] R^{2}(1+\cos \theta)^{2}}$ is the ratio between the normal and tangential wave-vector components for the longitudinal plasmons. In the limit $\beta \rightarrow 0$, $\gamma(\theta)$ diverges, and the local result is recovered [15]. Note though that, in general, $\alpha(\theta)$ and $\gamma(\theta)$ must be solved recursively.

The plasmonic behavior of a dimer of nonlocal touching nanowires is qualitatively different from the local prediction. In the local approximation, there is a continuous spectrum, and modes propagate towards the touching point but can never reach it. In the nonlocal case, the modes can sneak past the touching point and circulate round and

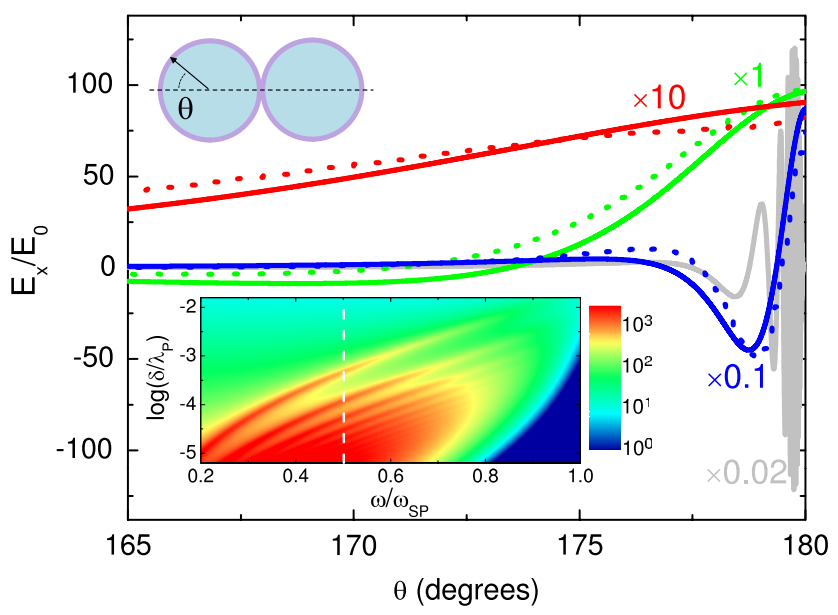

FIG. 2 (color online). Field enhancement in the vicinity of the touching point of two $10 \mathrm{~nm}$ radii Ag nanowires at $\omega=0.5 \omega_{\mathrm{SP}}$ and different degrees of nonlocality $\delta / \lambda_{P}: 10^{-4}$ (blue line), $10^{-3}$ (green line) - this is a realistic value for $\mathrm{Ag}$ - and $10^{-2}$ (red). Solid (dotted) lines show transformation-optics (numerical) results. The gray line plots $E_{x} / E_{0}$ under the local description. The inset displays $\left|E_{x} / E_{0}\right|$ at $\theta=180^{\circ}$ as a function of the incident frequency, $\omega / \omega_{\mathrm{SP}}$ and $\delta / \lambda_{P}$. The white dashed line indicates the operating frequency in the main panel. 
round the structure. This circulation leads to quantization of the modes and to a discrete spectrum. The amplitude modulation associated to this quantization is reflected in Eq. (2) through $f(\omega)=\left(1+e^{i \varphi(\omega)}\right)^{-1}$, where $\varphi(\omega)=\frac{1}{2} \times$ $\int_{0}^{2 \pi} \frac{d \theta^{\prime} \alpha\left(\theta^{\prime}\right)}{1+\cos \theta^{\prime}}$ is the phase accumulated by the SPPs in one loop around the nanowires. Finally, the radiative reaction term

$$
\eta(\omega)=\left|1+\frac{\pi^{2}}{2}\left(\frac{R \omega}{c}\right)^{2}\left\{\frac{1}{i \pi} \mathrm{Li}_{2}\left[e^{\alpha_{0}}\right]+2 \alpha_{0}[f(\omega)-1]\right\}\right|,
$$

where $\alpha_{0}=\alpha(\theta=0)$ and $\mathrm{Li}_{2}$ is the dilogarithm function, describes the interaction between the scattered fields and the nanowires dipole moment itself [12].

Figure 2 plots the field enhancement evaluated at $\omega=$ $0.5 \omega_{\mathrm{SP}}$ (where $\omega_{\mathrm{SP}}=0.94 \omega_{P}=3.67 \mathrm{eV}$ is the Ag surface plasmon frequency) close to the contact point between two $10 \mathrm{~nm}$ radii nanowires. Different degrees of nonlocality (colors), measured by the ratio $\beta / c=\delta / \lambda_{P}$ (where $\lambda_{P}=c / \omega_{P}$ ), are compared to the local result (gray). Solid lines correspond to the prediction from Eq. (2), whereas dotted lines render finite element COMSOL simulations [5] implementing the hydrodynamical permittivities above. Figure 2 shows that spatial dispersion in $\epsilon_{L}(\mathbf{k}, \omega)$ diminishes the ability of SPP modes to concentrate EM energy at the structure singularity, frustrating the drastic reduction that their group velocity and effective wavelength experience within the local description. However, our theoretical results demonstrate that, for realistic conditions $\delta=10^{-3} \lambda_{P}$ (green line), field enhancements as large as 2 orders of magnitude are obtained.

Figure 2 not only indicates that, as mentioned above, EM fields do not vanish in their propagation towards the dimer singularity but also reveals that $\left|\frac{E_{x}}{E_{0}}\right|$ is maximum at that position. The inset displays (in log scale) the absolute value of the field enhancement at $\theta=180^{\circ}$ as a function of the incident frequency and $\log \left(\delta / \lambda_{P}\right)$. It shows that the structure yields enhancement maxima larger than $10^{3}$. Importantly, the frequency at which these take place depends on $\delta / \lambda_{P}$, which shows that they are related to nonlocality. Note that, although not shown, $\left|\frac{E_{x}}{E_{0}}\right|=0$ at the touching point for $\delta \leq 10^{-8} \lambda_{P}$, in agreement with the local description.

In order to gain physical insight into the origin of the field enhancement maxima above, we look for their fingerprint in the absorption cross section. For this magnitude, our TO approach yields

$$
\sigma_{\mathrm{abs}}=\frac{(2 \pi R)^{2} \omega}{\eta^{2}(\omega) c} \operatorname{Re}\left\{\frac{1}{i \pi} \operatorname{Li}_{2}\left[e^{\alpha_{0}}\right]+2 \alpha_{0}[f(\omega)-1]\right\} .
$$

Figure 3(a) displays the absorption spectra for the $R=$ $10 \mathrm{~nm}$ nanowire dimers considered in Fig. 2. Solid lines plot the cross sections obtained from Eq. (5), and dots
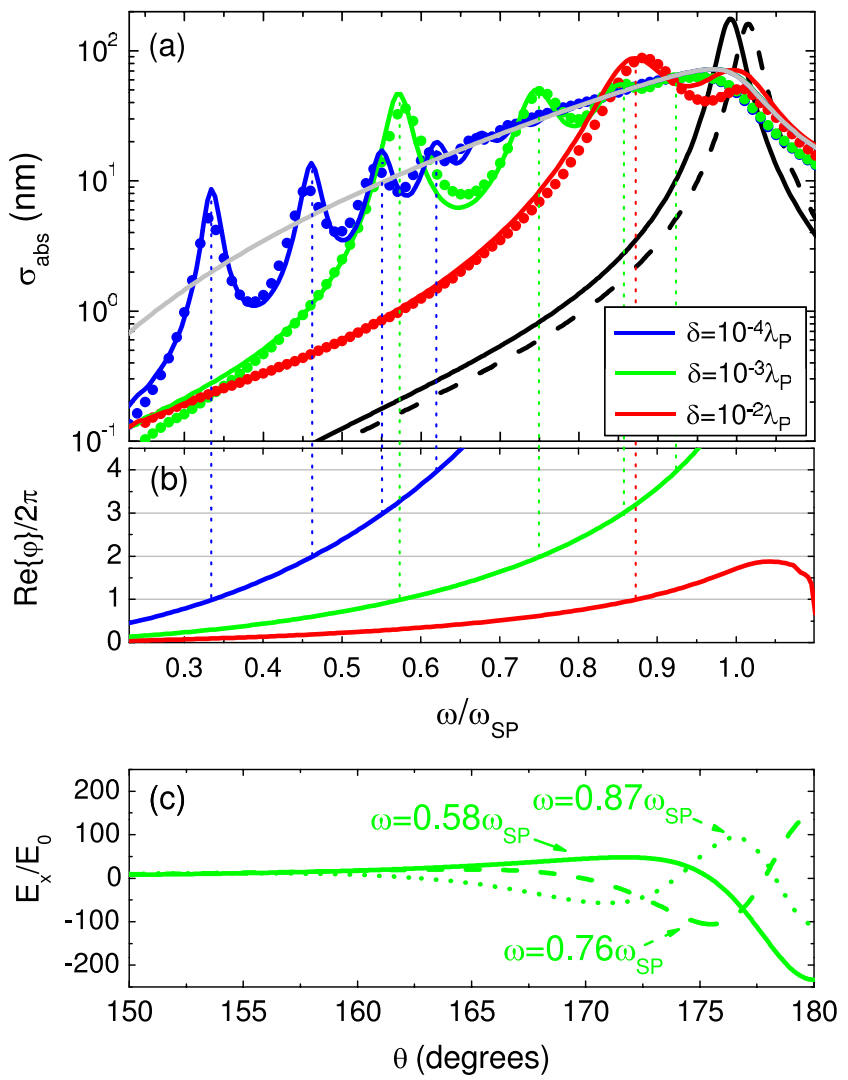

FIG. 3 (color online). (a) Theoretical (solid lines) and numerical (dots) absorption spectra for $10 \mathrm{~nm}$ radii nonlocal nanowires, together with the cross section obtained within the local approximation (gray dashed line). $\sigma_{\text {abs }}$ for a local (nonlocal, $\delta=10^{-2} \lambda_{P}$ ) single nanowire is also plotted as a solid (dashed) black line. (b) Real part of the phase accumulated by the circulating surface plasmon modes in one loop around the nanowires. (c) Field enhancement close to the touching point at the three lowest nonlocal resonances for $\delta=10^{-3} \lambda_{P}$ (value appropriate for silver).

render numerical results. As in the field enhancement calculations, the agreement between theory and simulations is remarkable. For comparison, $\sigma_{\text {abs }}$ for a local dimer (gray line) and local (solid black line) and nonlocal ( $\delta=$ $10^{-2} \lambda_{P}$, dashed line) single nanowires are also shown. Whereas spatial dispersion only blueshifts the dipolar resonance of isolated nanowires, its impact on the touching geometry is much more significant. Nonlocality gives rise to a set of absorption peaks whose position coincides with the field enhancement maxima in Fig. 2. The number of these nonlocal resonances diminishes with increasing $\beta$, as they shift to higher frequencies in a similar manner as the dipolar peak in the single nanowire spectrum. Importantly, Fig. 3(a) shows that the broadband response of touching dimers is lost at frequencies below the lowest nonlocal resonance.

Figure 3(b) renders the real part of $\varphi(\omega)$ for the three nonlocal dimers in Fig. 3(a). The spectral position of the 

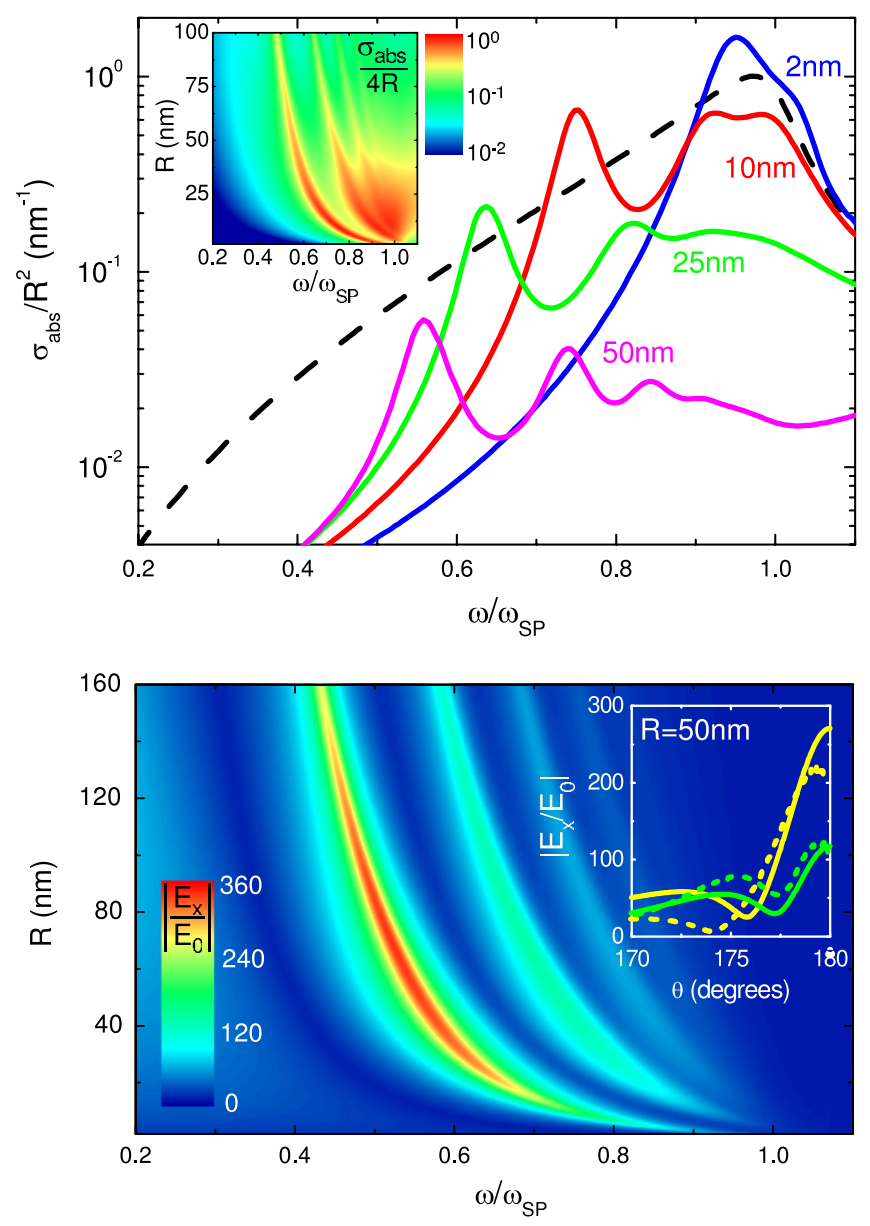

FIG. 4 (color online). Upper panel: Absorption cross section normalized to the structure area for $\operatorname{Ag}\left(\beta=3.6 \times 10^{-3} c\right)$ nanowires of different radius. Color solid lines plot $\sigma_{\text {abs }} / R^{2}$ evaluated from Eq. (5), and the dashed black line renders the electrostatic local result for all $R$. The inset shows the absorption efficiency versus the incident frequency and nanowires radius. Lower panel: Absolute value of the field enhancement at the touching point as a function of $\omega / \omega_{\mathrm{SP}}$ and $R$. Solid (dashed) lines in the inset render theoretical (numerical) $\left|E_{x} / E_{0}\right|$ at the two lowest enhancement maxima for $R=50 \mathrm{~nm}$ : $0.55 \omega_{\mathrm{SP}}$ (yellow) and $0.72 \omega_{\mathrm{SP}}$ (green).

cross section maxima coincide with the condition $\varphi(\omega)=$ $2 \pi N$ (where $N$ is an integer number). This demonstrates that they originate from the constructive superposition of the SPP modes circulating around the nanowires. Figure 3(c) plots the field enhancement versus $\theta$ evaluated at the three lowest absorption maxima for a value of $\delta$ appropriate to silver, $\delta=10^{-3} \lambda_{P}$ [green line in Fig. 3(a)]. In analogy with separated local nanowires [16], the integer $N$ above can be related to the number of nodes that the SPP fields present near the singularity.

Finally, we use our TO approach to explore the interplay between radiative losses and nonlocality in touching $\mathrm{Ag}$ nanowires. Although these effects have different origins, their impact on the optical response of the nanostructure is measured by its physical size. Nonlocality is important for small structures, radiative effects for large ones. Both reduce the enhancement of fields. In accordance with the literature [10], we take $\beta=3.6 \times 10^{-3} c$, which corresponds to $\delta=0.16 \mathrm{~nm}$ (note the good agreement with $\delta_{C}$ above). The upper panel of Fig. 4 renders the absorption spectra normalized to $R^{2}$ for various nanowire sizes (color solid lines). Within the electrostatic local approximation [15], $\sigma_{\text {abs }} / R^{2}$ (black dashed line) coincides for all the structures. The comparison between Eq. (5) and the electrostatic local prediction clarifies how nonlocality and radiation losses modify the optical properties of the system. For small sizes $(R=2 \mathrm{~nm})$, radiation is negligible, but the weight of spatial dispersion (measured by the factor $\delta / R)$ is high. This leads to a single narrow maximum in $\sigma_{\text {abs }} / R^{2}$ that resembles the behavior of individual nanowires [see Fig. 3(a)]. For larger sizes, the spectra are lowered due to radiative reaction, while the impact of nonlocality is diminished. Thus, the dimers interact less efficiently with the incoming light but within a broader frequency window. In the inset, the absorption efficiency $\left(\sigma_{\mathrm{abs}} / 4 R\right)$ is rendered as a function of $\omega / \omega_{\mathrm{SP}}$ and the nanowire radius. It shows that the light collection performance of the structure is optimized for nanowire radii between 5 and $50 \mathrm{~nm}$, where the compromise between radiative and nonlocal effects is most convenient.

The lower panel of Fig. 4 renders $\left|\frac{E_{x}}{E_{0}}\right|$ at the touching point as a function of the incident frequency and $R$. Importantly, whereas the shrinking of the structure always improves its ability to concentrate light in the local description [12], the inclusion of nonlocal effects truncates this trend. Figure 4 indicates that the lowest nonlocal resonance governs the field enhancement behavior of the system. It also shows that the balance between radiation losses and nonlocal effects leads to maximum field enhancements for $R$ between 25 and $80 \mathrm{~nm}$. For such nanowire sizes, $\left|\frac{E_{x}}{E_{0}}\right| \geq 300$ at resonance, yielding intensity factors as large as $10^{5}$. This demonstrates that $\mathrm{Ag}$ touching nanowires feature prominent light-harvesting capabilities despite the adverse consequences that radiation and nonlocal effects have on them. The inset in Fig. 4 plots theoretical (numerical) $\left|\frac{E_{x}}{E_{0}}\right|$ in the vicinity of the dimer singularity evaluated at the two lowest resonances for $R=50 \mathrm{~nm}$. It proves the accuracy of our theory for $\sim 100 \mathrm{~nm}$ radii nanowires and validates our analysis of the dependence of their optical properties on the nanostructure size.

In conclusion, we have presented an elegant transformation-optics approach that makes possible the quasianalytical study of nonlocal effects in the plasmonic properties of metal nanoparticles. We have focused on dimers of touching nanowires, showing that spatial dispersion leads to the formation of nonlocal resonances in this geometry. These are caused by the circulation of surface plasmon modes around the nanowires, which trespass the 
structure singularity and alter significantly its optical response. Finally, we have investigated radiative and nonlocal effects in realistic Ag dimers, demonstrating the robustness of their prominent light-harvesting capabilities and providing an optimum size for the nanostructure.

The authors thank A. P. Horsfield for useful discussions. This work was supported by the ESF plasmonbionanosense program, the Leverhulme Trust, and the Engineering and Physical Sciences Research Council (EPSRC).

[1] S. A. Maier, Plasmonics: Fundamentals and Applications (Springer-Verlag, New York, 2007).

[2] C. Kittel, Introduction to Solid State Physics (Wiley, New York, 1996).

[3] A. R. Melnyk and M. J. Harrison, Phys. Rev. B 2, 835 (1970); G. Agarwal et al., Phys. Rev. B 10, 1447 (1974); G. C. Aers, A. D. Boardman, and P. A. Young, J. Phys. C 10, 2437 (1977).

[4] J. M. McMahon, S. K. Gray, and G. C. Schatz, Phys. Rev. Lett. 103, 097403 (2009).
[5] S. Raza et al., Phys. Rev. B 84, 121412(R) (2011); G. Toscano et al., Proc. SPIE Int. Soc. Opt. Eng. 7757, $77571 \mathrm{~T}$ (2010).

[6] C. David and F. J. García de Abajo, J. Phys. Chem. C 115, 19470 (2011).

[7] R. Fuchs and F. Claro, Phys. Rev. B 35, 3722 (1987); R. Rojas, F. Claro, and R. Fuchs, Phys. Rev. B 37, 6799 (1988).

[8] R. Ruppin, Opt. Commun. 190, 205 (2001).

[9] A. Aubry et al., Nano Lett. 10, 2574 (2010); A.I. Fernández-Domínguez, S. A. Maier, and J.B. Pendry, Phys. Rev. Lett. 105, 266807 (2010).

[10] A. D. Boardman, Electromagnetic Surface Modes (Wiley, New York, 1982).

[11] E. D. Palik, Handbook of Optical Constants of Solids (Academic, New York, 1985).

[12] A. Aubry et al., Phys. Rev. B 82, 205109 (2010).

[13] J. Zuloaga et al., Nano Lett. 9, 887 (2009); , ACS Nano 4, 5269 (2010).

[14] O. Perez-Gonzalez et al., Nano Lett. 10, 3090 (2010).

[15] D. Y. Lei et al., New J. Phys. 12, 093030 (2010).

[16] A. Aubry et al., Phys. Rev. Lett. 105, 233901 (2010). 